\title{
Stereotactic radiation therapy for oligometastases or oligorecurrence within mediastinal lymph nodes
}

\author{
Huan-Huan Wang ${ }^{1}$, Nicholas G. Zaorsky ${ }^{2}$, Mao-Bin Meng ${ }^{1}$, Xian-Liang Zeng ${ }^{1}$, Lei \\ Deng $^{3}$, Yong-Chun Song ${ }^{1}$, Hong-Qing Zhuang ${ }^{1}$, Feng-Tong Li ${ }^{1}$, Lu-Jun Zhao ${ }^{1}$, Zhi- \\ Yong Yuan ${ }^{1}$, Ping Wang ${ }^{1}$, Xi-Shan $\mathrm{Hao}^{4}$ \\ 1 Department of Radiation Oncology, CyberKnife Center, and Key Laboratory of Cancer Prevention and Therapy, Tianjin \\ Medical University Cancer Institute \& Hospital, National Clinical Research Center for Cancer, Tianjin, China \\ 2 Department of Radiation Oncology, Fox Chase Cancer Center, Philadelphia, PA, USA \\ ${ }^{3}$ Department of Thoracic Cancer and Huaxi Student Society of Oncology Research, West China Hospital, West China School \\ of Medicine, Sichuan University, Chengdu, China \\ ${ }^{4}$ Department of Gastrointestinal Surgery, Key Laboratory of Cancer Prevention and Therapy, National Clinical Research \\ Center for Cancer, Tianjin Medical University Cancer Institute \& Hospital, Tianjin, China
}

Correspondence to: Mao-Bin Meng, email: doctormm991@hotmail.com

Keywords: stereotactic radiation therapy, mediastinal lymph node, neoplasm metastasis, oligometastasis, oligorecurrence Received: October 26, $2015 \quad$ Accepted: February 11, 2016

Published: February 23, 2016

\section{ABSTRACT}

Aims: This study evaluated the safety and efficacy of stereotactic radiation therapy (SRT) for the treatment of patients with oligometastases or oligorecurrence within mediastinal lymph nodes (MLNs) originating from different tumors.

Methods: Between October 2006 and May 2015, patients with MLN oligometastases or oligorecurrence were enrolled and treated with SRT at our hospital. The primary endpoint was MLN local control (LC). Secondary endpoints were time to symptom alleviation, overall survival (OS) after SRT, and toxicity using the Common Terminology Criteria for Adverse Events (CTCAE v4.0).

Results: Eighty-five patients with 98 MLN oligometastases or oligorecurrences were treated with SRT. For the entire cohort, the 1-year and 5-year actuarial LC rates were $97 \%$ and $77 \%$, respectively. Of 53 symptomatic patients, symptom alleviation was observed in 47 (89\%) after a median of 5 days (range, 3-30 days). The median OS was $\mathbf{2 7 . 2}$ months for all patients. For patients with non-small cell lung cancer, univariate and multivariate analyses revealed that a shorter interval between diagnosis of primary tumors and SRT and larger MLN SRT volume were associated with worse OS. CTCAE v4.0 $\geq$ Grade 3 toxicities occurred in six patients (7\%), with Grade 5 in three patients (all with RT history to MLN station 7).

Conclusions: SRT is a safe and efficacious treatment modality for patients with oligometastases or oligorecurrence to MLNs originating from different tumors, except for patients who received radiotherapy to MLN station 7. Further investigation is warranted to identify the patients who benefit most from this treatment modality.

\section{INTRODUCTION}

Oligometastasis and oligorecurrence refer to a state in which a patient has a limited number of distant metastatic regions (typically $\leq 5$ ) that contain the primary tumor. These states may be noted at the time of diagnosis (i.e. oligometastsis) or as failure after definitive therapy (i.e. oligorecurrence). Although most patients with distant metastases are typically incurable, the oligometastatic/ oligorecurrent state implies that metastasis-directed therapy may cure the disease (e.g. liver metastasis from colorectal cancer) [1].

As of 2016, there is no standard approach for managing patients with oligometastases or oligorecurrence. Conventional therapy for those with oligometastatic/recurrent disease is systemic therapy alone (e.g. with chemotherapy, hormones, or targeted agents); unfortunately, this rarely eradicates gross disease. Moreover, surgical salvage of these patients is not always feasible, given their proclivity to juxtapose critical 
structures (e.g. esophagus, great vessels, and trachea) [2].

Over the last decade, evidence has emerged suggesting patients with oligometastases or oligorecurrence may be cured with metastasis-directed stereotactic radiation therapy (SRT) [3-10]. SRT is a type of external beam radiation therapy (EBRT) that delivers RT accurately and precisely to the tumor, with fewer fractions and higher biologically equivalent dose (BED) than conventionally fractionated radiation therapy. SRT is divided into stereotactic body RT (SBRT; the delivery of 3.5-15 Gy per fraction, in 5 fractions or less) and fractionated stereotactic RT (FSRT; with delivery in more than 5 fractions). SRT can be delivered using either a traditional linear accelerator or a robotic arm (i.e. CyberKnife).

Our preliminary report on SRT for recurrent/ secondary primary mediastinal lymph node (MLN) metastases from non-small cell lung cancer (NSCLC) revealed that SRT appears to be a safe and efficacious treatment modality for patients without previous RT [11]. The purpose of the current study is to update our previous report and evaluate the safety and efficacy of SRT for patients with oligometastases or oligorecurrence to MLNs originating from different tumors.

\section{RESULTS}

\section{Patient and treatment characteristics}

Clinical information on 3,332 patients with different primary or metastatic tumors treated with SRT between October 1, 2006 and May 1, 2015 at the CyberKnife Center of Tianjin Medical University Cancer Institute \& Hospital was reviewed. Of all patients, there were85 patients with 98 MLN oligometastases or oligorecurrences treated with SRT. The median interval from the diagnosis of primary cancer to the first day of SRT was 21.2 months (range, 0.5 - 305.7 months). Among the patients, 72 had one MLN oligometastasis or oligorecurrence, and thirteen patients had two MLN oligometastases or oligorecurrences within one MLN zone. Thirty-seven patients (37/85, 44\%) had synchronous oligometastases or oligorecurrence, and localized treatment was applied to all lesions.

The patient and treatment characteristics of all patients and NSCLC patients are shown in Table 1 and Supplementary file 2, respectively. The radiation doses to normal organs (e.g. trachea, esophagus, aorta) are shown in Supplementary file 3. An example of successful treatment plan is shown in Figure 1. A treatment plan where the patient experienced Grade 5 toxicity (despite meeting dose constraints) is shown in Figure 2. Table 2 provides a detailed summary of the MLN stations and SRT treatment planning parameters for all patients. Figure 3 shows the relationships of various SRT parameters.

\section{MLN response and time to symptoms alleviation}

Clinical tumor responses were evaluated at 6 months after SRT using CT and/or PET-CT scans. Out of the 85 patients, $74(74 / 85,87 \%)$ had a CR, five $(5 / 85,6 \%)$ had PR, three $(3 / 85,4 \%)$ had SD, and three $(3 / 85,4 \%)$ had PD. The 1-year and 5-year actuarial LC rates for all eligible patients were $97 \%$ and $77 \%$, respectively (Figure 4A). Symptom alleviation was observed in 47 out of 53 patients (89\%) after a median of 5 days (range, 3-30 days), lasting through the follow-up period. Of these 47 patients, there was complete resolution of symptoms and discontinuation of medications in 42 patients.

\section{Overall survival}

For the whole cohort, median follow-up was 42.2 months (range, 8.1-389.9 months). The median OS from diagnosis and from SRT, respectively, was: 53.0 months and 27.2 months for all patients (Figure 4B); 52.3 months and 32.2 months for NSCLC (Figure 4C).

The following analyses were carried out for the 53 NSCLC patients only, since they made up the majority of the primary tumors. Further characteristics of these patients are listed in Supplementary File 2. The 1-, 3 -, and 5-year OS rates from SRT were 78.2\%, 43.6\%, and $21.3 \%$, respectively. A worse OS was associated with a shorter time until SRT, larger PTV size, or nonuse of chemotherapy (Figure 4D). Differences in OS from SRT were insignificant for presence vs. absence of symptoms $(p=0.15)$, synchronicity of oligometastases or oligorecurrence $(p=0.26)$, number of lesions ( $p=$ $0.25)$, MLN location of lesions $(p=0.48)$, SRT treatment intent $(p=0.73)$, and patient history of RT $(p=0.12)$. On multivariate analysis (Table 3 ) for NSCLC patients, a worse OS was noted for patients with shorter interval time between diagnosis of primary tumors and SRT (median, 13.6 months) and a larger MLN PTV (median, $15.3 \mathrm{~mL}$ ).

\section{Patterns of failure}

Among all eligible patients three (3/85, 4\%) relapsed within the PTV. These patients had disease in MLNs of 2R, 10R, or 1L. Forty-nine patients (49/85, 58\%) had out-of-field progression with a median of 9.6 months after SRT (range, 0.5-85.6 months); 33 patients (33/85, $39 \%$ ) had no progression after SRT. Among the patients with progression, two $(2 / 85,2 \%)$ had diffuse progression including regional failure. These two patients had distant metastases to lung, bone, brain, liver and non-regional lymph nodes. 

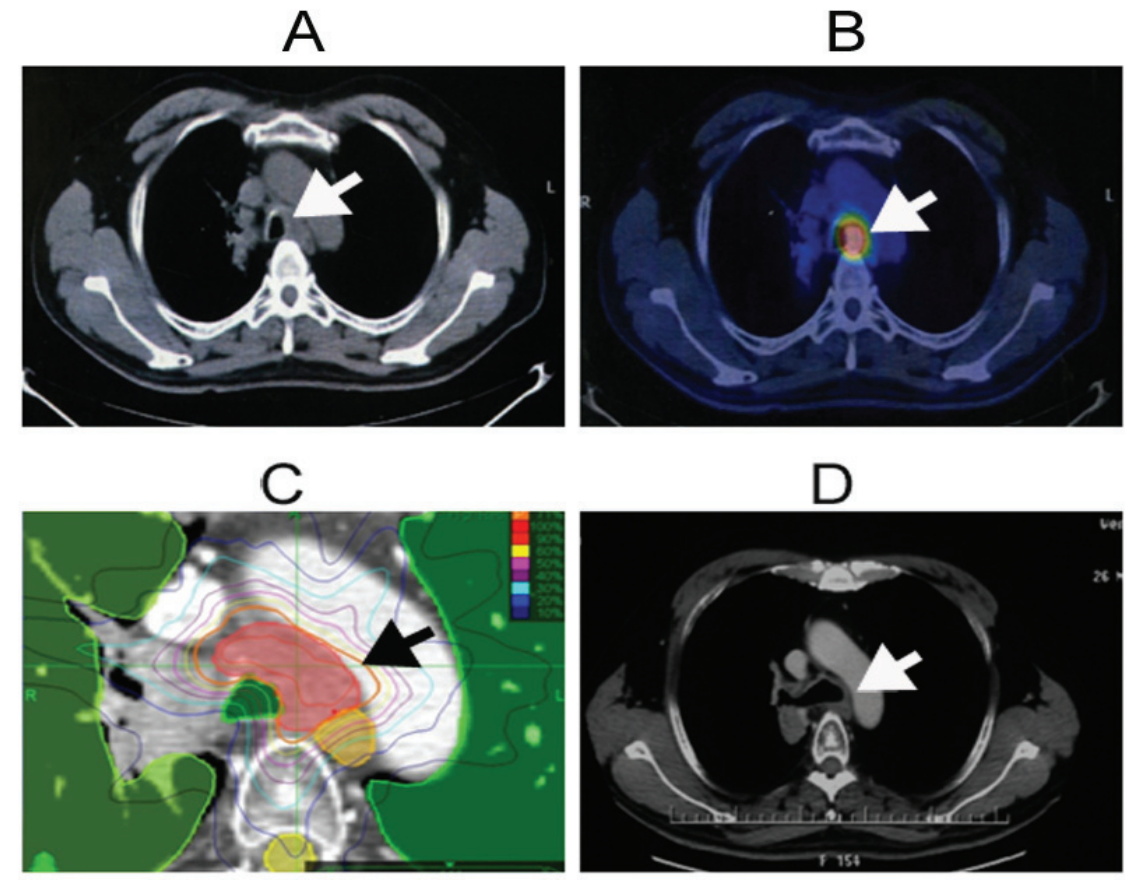

Figure 1: An illustrative case of successful SRT for oligometastatic NSCLC to a MLN. 52-year-old man with squamous cell carcinoma in the upper lobe of the left lung. Images were taken after surgery and systematic lymphadenectomy; he was treated for a station 4 MLN oligorecurrence with SRT prescribed at 48 Gy in 8 fractions. The pretreatment CT A. and PET-CT B. showed the hyperactive metabolic activity in the station 4 MLN oligorecurrence. C. The planning CT and isodose distributions with SRT and contours depict planning target volume (red), lung (green), esophagus and cord (yellow), and bronchus (green). D. Post-SRT CT showed complete response. MLN: mediastinal lymph node; SRT: stereotactic radiation therapy; CT: computed tomography; PET-CT: positron emission tomography- computed tomography. Note: The arrows indicate MLN oligorecurrence.

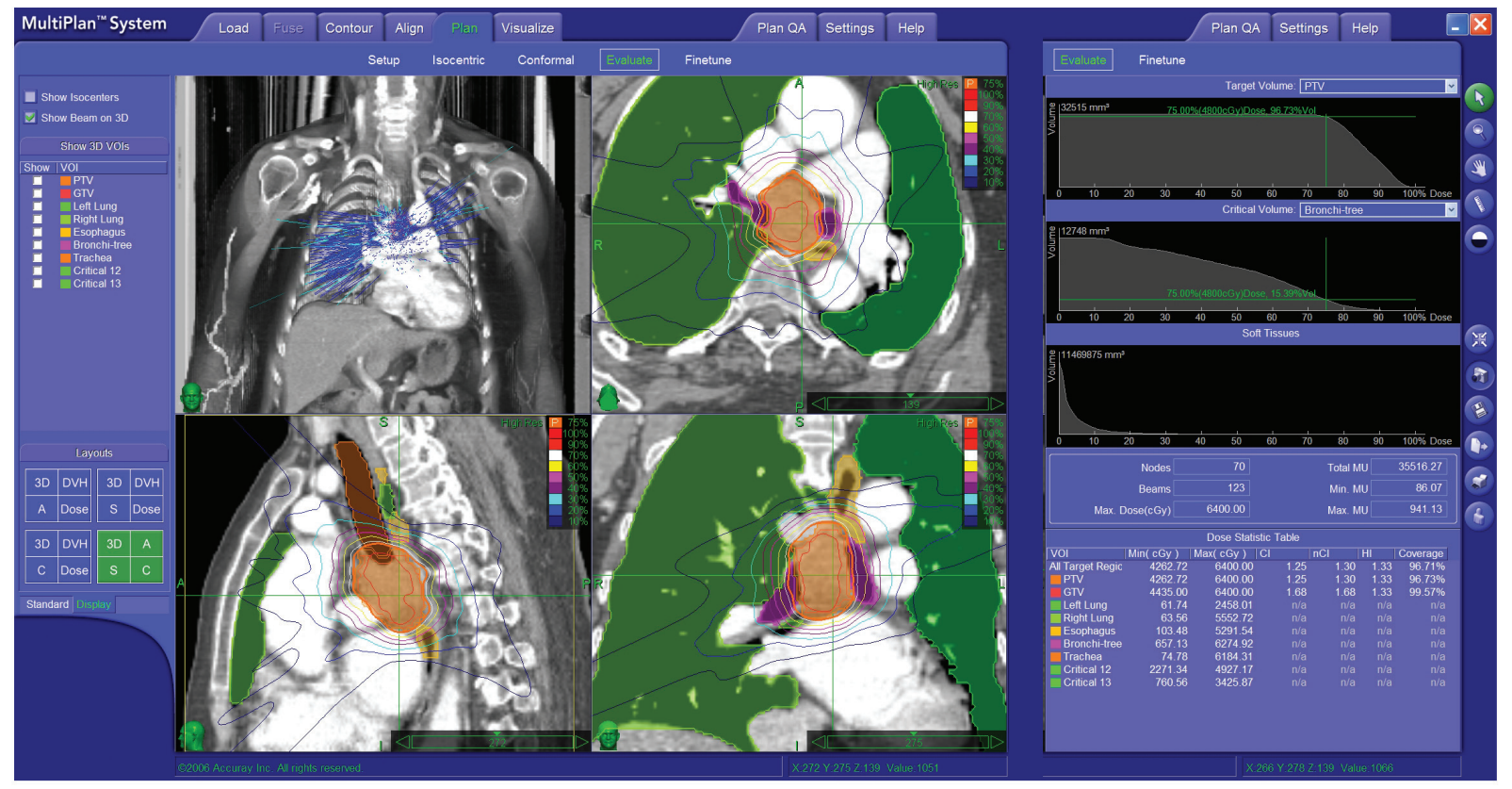

Figure 2: An illustrative case of Grade 5 toxicity after SRT for oligometastatic NSCLC to a station 7 MLN. This patient, a 64-year-old woman with squamous cell lung cancer located in her left lower lobe with station 7 MLN, received SRT 6.8 months after completion of definitive RT. SRT was 48 Gy in 8 fractions, prescribed to the $75 \%$ isodose line, which covered $95 \%$ of the PTV. The PTV was also amended to avoid adjacent organs at risk (i.e. esophagus, brachial, trachea, spine cord, and heart). The outermost line is the $30 \%$ isodose line (outermost blue line). Unfortunately, the patient died from a tracheoesophageal fistula six weeks after completion of SRT, despite meeting dose constraints. MLN: mediastinal lymph node; RT: radiation therapy; SRT: stereotactic radiation therapy; Gy: Gray; GTV: gross tumor volume; CT: computed tomography; PTV: planning target volume. 


\section{Toxicities}

Toxicity of all patients and the radiation dose for trachea, esophagus, heart, aorta, and brachial plexus are summarized in Table 4 and Supplementary file 3, respectively. Eleven patients $(11 / 85,13 \%)$ experienced CTCAE v4.0 Grade 1 to 2 acute toxicities. Four patients (4/85, 5\%) experienced Grade 3 pneumonitis, esophagitis, and/or tracheitis; these Grade 3 toxicities were generally transient and resolved with conservative management. Late radiation toxicities were observed in six patients $(6 / 85,7 \%)$; three of them $(3 / 85,4 \%)$ died from Grade 5 late radiation toxicities (either tracheoesophageal or esophageal-mediastinal fistula), all of which had history of RT to MLNs in station 7.

\section{DISCUSSION}

To our knowledge, this is the largest study to evaluate the safety and efficacy of SRT for patients with oligometastases or oligorecurrences of various tumors to MLNs. Our results demonstrate that SRT is a safe and efficacious treatment modality for such patients, except for those who had history of RT to MLN station 7. Further investigation is warranted to identify the patients who benefit most from this treatment modality.

MLN oligometastasis and oligorecurrence are common in NSCLC, occurring in $20 \%$ of patients with stage I disease and up to $50 \%$ of patients with stage III disease. The majority of failures are confined to the thorax, and survival after recurrence is $<30 \%$ [1213]. SRT appears to be a promising treatment option for most patients [14-18] (Figures 1 and 2). In this study, the majority of patients $(53 / 85,62 \%)$ had MLN oligometastases or oligorecurrence from NSCLC (Table 1 ), and we performed a subset analyses of these patients. Notably, more than $30 \%$ of patients had oligorecurrence in MLNs typically inaccessible by minimally invasive techniques (e.g. levels 2-7), but allowing achievement of high $\mathrm{BED}_{10}$ s, typically $>100$ Gy (Table 2 ).

Our results are consistent with previous studies which suggest that worse OS is associated with short interval since previous RT, poor patient performance status, large target volume, and previous RT to the adjacent critical structures (Figure 3) [11, 19-29]. However, we did not note worse LC with larger PTVs, as seen in other studies $[20,28]$, perhaps because we were generally able to maintain $\mathrm{BED}_{10} \mathrm{~s}>100$ (Table 2). The 1-year and 5 -year actuarial LC rates for all eligible patients $(97 \%$ and $77 \%$, respectively) were higher than reported rates for conventional RT (typically $<65 \%$ at 2 years) [30].

The median OS for our NSCLC patients (32 months) is superior to the OS of those treated with conventional RT (11 - 19 months) [31] and of unresectable IIIA and IIIB NSCLC patients receiving concurrent chemo-RT (16 - 19 months) [32-34]. We hypothesize that the longer OS is due to a longer cell replication time (as suggested by
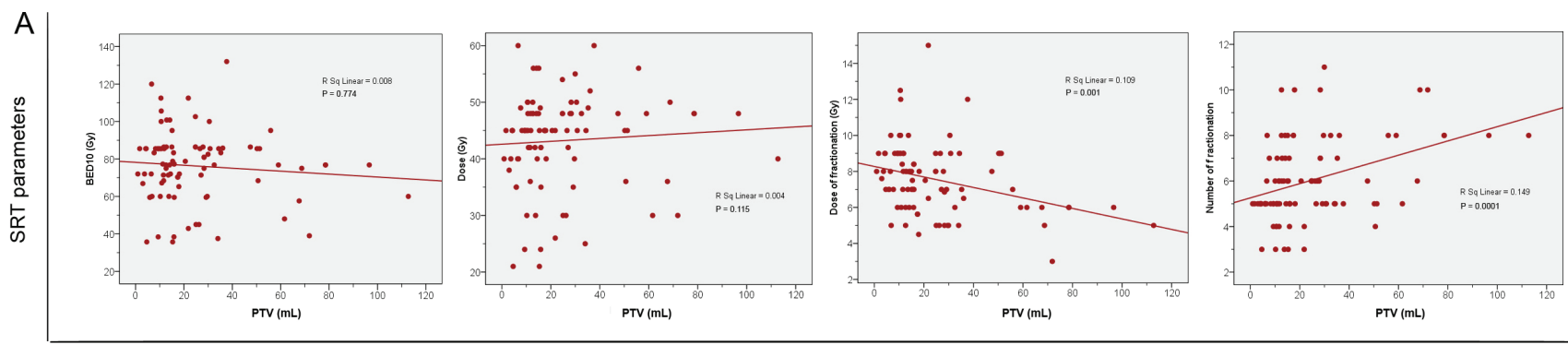

$\operatorname{PTV}(\mathrm{mL})$
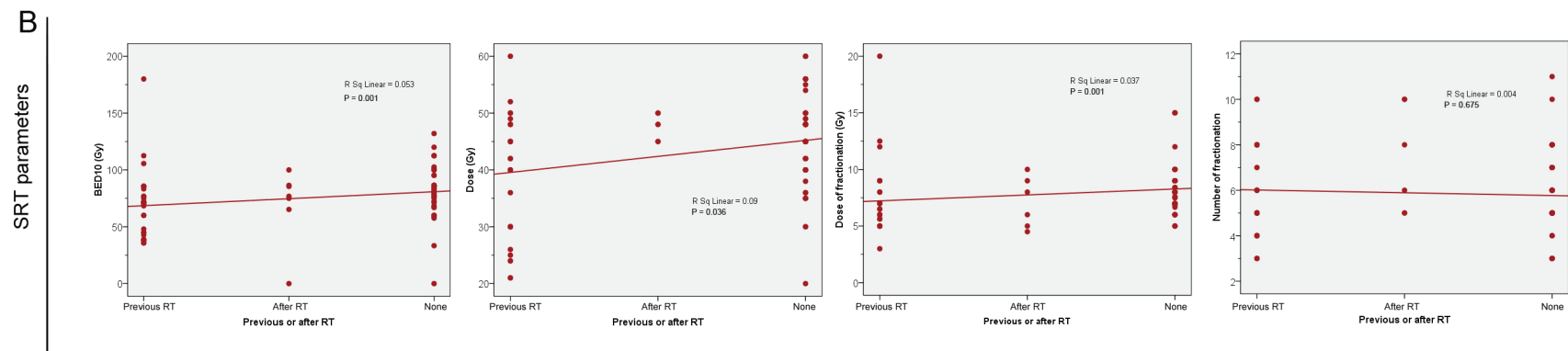

History of RT (overlap of the present lesions with the field of RT)

Figure 3: Scatterplots of: A. The relationship between SRT parameters and MLN PTV. B. The relationship between SRT parameters and patients with history of RT. Some patients first received SRT and then received conventionally fractionated RT; others first received conventionally fractionated RT and then received SRT; others received SRT alone. SRT: stereotactic radiation therapy; RT: radiation therapy; BED: biologically equivalent dose. 
Table 1: Summary of patient and treatment characteristics $(N=85)$

\begin{tabular}{|c|c|}
\hline \begin{tabular}{|l|} 
Parameter \\
\end{tabular} & No. (\%) \\
\hline \multicolumn{2}{|l|}{ Age (years) at the SRT } \\
\hline \begin{tabular}{|l} 
Median (range) \\
\end{tabular} & $59(32-89)$ \\
\hline \multicolumn{2}{|l|}{ Gender } \\
\hline Male & $54(64)$ \\
\hline Female & $31(36)$ \\
\hline \multicolumn{2}{|l|}{\begin{tabular}{|l|} 
Primary tumors \\
\end{tabular}} \\
\hline Non-small cell lung cancer & $53(62)$ \\
\hline Esophageal carcinoma & $7(8)$ \\
\hline Breast cancer & $7(8)$ \\
\hline Hepatocellular carcinoma & $3(4)$ \\
\hline Thyroid carcinoma & $3(4)$ \\
\hline Kidney cancer & $3(4)$ \\
\hline Bladder cancer & $2(2)$ \\
\hline Thymic carcinoma & $1(1)$ \\
\hline Nasopharyngeal cancer & $1(1)$ \\
\hline Ovarian cancer & $1(1)$ \\
\hline Rectal cancer & $1(1)$ \\
\hline Cervical cancer & $1(1)$ \\
\hline Sublingual adenocarcinoma & $1(1)$ \\
\hline Submandibular gland cancer & $1(1)$ \\
\hline \multicolumn{2}{|l|}{ No. of MLNs within LN zone } \\
\hline 1 & $72(85)$ \\
\hline 2 & $13(15)$ \\
\hline \multicolumn{2}{|l|}{ Clinical symptoms of MLNs } \\
\hline Yes & $53(62)$ \\
\hline No & $32(38)$ \\
\hline \multicolumn{2}{|l|}{ Radiographic diagnosis of MLNs } \\
\hline PET-CT & $64(75)$ \\
\hline $\mathrm{CT}$ & $21(25)$ \\
\hline \multicolumn{2}{|l|}{ Synchronous metastases } \\
\hline Yes & $37(44)$ \\
\hline No & $48(25)$ \\
\hline \multicolumn{2}{|c|}{ Interval between the diagnosis of primary and SRT (median, in months) } \\
\hline$<21.23$ & $49(58)$ \\
\hline$\geq 21.23$ & $36(42)$ \\
\hline \multicolumn{2}{|c|}{ History ofRT (overlap of the present lesions with the field of RT) } \\
\hline Yes & $29(34)$ \\
\hline No & $56(66)$ \\
\hline \multicolumn{2}{|l|}{ SRT treatment intent } \\
\hline \begin{tabular}{|l|} 
Curative \\
\end{tabular} & $83(98)$ \\
\hline Palliative & $2(3)$ \\
\hline \multicolumn{2}{|l|}{ Other treatments after SRT } \\
\hline Chemotherapy & $37(48)$ \\
\hline Endocrine therapy ${ }^{\dagger}$ & $3(4)$ \\
\hline Molecular targeted therapy* & $9(11)$ \\
\hline
\end{tabular}

Three breast cancer patients with positive of ER, PR, and Her-2 received endocrine therapy.

$\star$ Seven lung adenocarcinoma patients with EGFR mutation received erlotinib or gefitinib, and 2 hepatocellular carcinoma received sorafenib.

Abbreviations: MLNs: mediastinal lymph nodes; PET-CT: positron emission tomography/computed tomography; CT: computed tomography; SRT: stereotactic radiation therapy; RT: radiotherapy. 
Table 2: MLN stations and SRT treatment parameters

\begin{tabular}{|c|c|c|c|c|c|c|c|}
\hline Nodal zone & No. $(\%) \dagger$ & PTV (mL) & $\begin{array}{l}\text { Prescription } \\
\text { dose (Gy) }\end{array}$ & $\begin{array}{l}\text { No. of } \\
\text { fractions }\end{array}$ & $\begin{array}{l}\text { Dose per } \\
\text { fraction (Gy) }\end{array}$ & BED10 (Gy) & $\begin{array}{l}\text { Prescription } \\
\text { isodose line } \\
(\%)\end{array}$ \\
\hline Upper & $46(47 \%)$ & & & & & & \\
\hline $1 \mathrm{R}$ & $5(11 \%)$ & \begin{tabular}{ll|}
25.9 & $(13.5-$ \\
$28.3)$
\end{tabular} & 45 (42-48) & $6(3-7)$ & $7(6.86-15)$ & $\begin{array}{ll}80.9 & (71.4- \\
112.5)\end{array}$ & $70(65-75)$ \\
\hline $1 \mathrm{~L}$ & $4(9 \%)$ & $27.0(3.0-34.0)$ & $36.5(25-48)$ & $5(5-6)$ & $7.3(5-8)$ & $\begin{array}{ll}73.0 & (37.5- \\
86.4)\end{array}$ & $78.5(65-81)$ \\
\hline $2 \mathrm{R}$ & $14(30 \%)$ & $16.0(0.9-68.7)$ & $45(21-50)$ & $5.5(3-10)$ & $6.93(5-10)$ & $72(35.7-100)$ & $73.5(62-81)$ \\
\hline $2 \mathrm{~L}$ & $3(7 \%)$ & $\begin{array}{|ll|}31.3 & (26.6- \\
36.0) & \\
\end{array}$ & $45(45-52)$ & $5(5-8)$ & $9(6.5-9)$ & $\begin{array}{ll}85.5 & (85.5- \\
85.8) & \\
\end{array}$ & $67.5(63-72)$ \\
\hline $3 \mathrm{~A}$ & $6(13 \%)$ & $\begin{array}{ll}34.3 & (13.8- \\
51.3) & \\
\end{array}$ & $45(30-60)$ & $5(3-5)$ & $9(8-12)$ & $85.5(60-132)$ & $79.5(76-82)$ \\
\hline $4 \mathrm{R}$ & $8(17 \%)$ & $11.5(4.4-50.6)$ & $45(36-50)$ & $5(3-6)$ & $9(7-15)$ & $\begin{array}{ll}85.5 & (68- \\
112.5) & \\
\end{array}$ & $76(70-81)$ \\
\hline $4 \mathrm{~L}$ & $6(13 \%)$ & $14.9(5.9-18.1)$ & 45 (35-49) & $5.5(5-8)$ & $7.5(6-8.4)$ & $\begin{array}{ll}77.0 & (59.5- \\
86.4) & \\
\end{array}$ & $71.5(66-74)$ \\
\hline Aorticopulmonary & $21(21 \%)$ & & & & & & \\
\hline 5 & $17(81 \%)$ & $\begin{array}{|ll|}13.3 & (1.7- \\
112.8)\end{array}$ & $42(24-50)$ & $5(3-10)$ & $8.4(4.5-10)$ & \begin{tabular}{|ll}
76.8 & $(38.4-$ \\
$100)$ & \\
\end{tabular} & $74(67-81)$ \\
\hline 6 & $4(19 \%)$ & $\begin{array}{ll}18.6 \\
34.4)\end{array}$ & 45 (21-45) & $4(3-6)$ & $8.25(7-15)$ & $\begin{array}{l}82.1 \\
112.5)\end{array}$ & $73.5(63-79)$ \\
\hline Subcarinal & $9(9 \%)$ & & & & & & \\
\hline 7 & $9(100 \%)$ & $12.6(7.7-71.8)$ & 45 (24-50) & $7(4-10)$ & $6(3-9)$ & $\begin{array}{|ll|}76.8 & (38.4- \\
85.5) & \\
\end{array}$ & $74(66-82)$ \\
\hline Lower & $7(7 \%)$ & & & & & & \\
\hline 8 & $2(29 \%)$ & $\begin{array}{ll}19.4 \\
28.3)\end{array}$ & $50(50,50)$ & $7(4,10)$ & $8.75(5,12.5)$ & \begin{tabular}{|ll}
93.75 & $(75$, \\
$112.5)$ &
\end{tabular} & $79.5(79,80)$ \\
\hline 9 & $5(71 \%)$ & $18.4(6.8-78.5$ & $45(30-60)$ & $6(3-8)$ & $6(5-20)$ & $76.8(45-180)$ & $77.5(77-80)$ \\
\hline Hilar-interlobar & $15(15 \%)$ & & & & & & \\
\hline $10 \mathrm{R}$ & $8(53 \%)$ & $\begin{array}{ll}26.3 & (10.6- \\
67.6) & \\
\end{array}$ & $52(36-56)$ & $6(4-8)$ & $8(6-12)$ & $\begin{array}{l}100.4 \quad(57.6- \\
105.6)\end{array}$ & $75(65-78)$ \\
\hline $10 \mathrm{~L}$ & $7(47 \%)$ & $35.2(6.6-96.6)$ & $49(30-60)$ & $7(5-11)$ & $7(5-10)$ & $83.3(48-120)$ & $76(70-82)$ \\
\hline All & $98(100 \%)$ & & & & & & \\
\hline
\end{tabular}

'Number of MLN metastases.

Abbreviations: MLNs: mediastinal lymph nodes; R: right; L: left. SRT: stereotactic radiation therapy; PTV: planning target

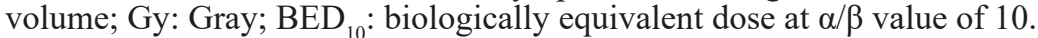

Note: all numbers presented are medians (with range or percent in parenthesis).

Table 3: Multivariate subgroup analyses for OS from SRT in NSCLC

\begin{tabular}{|l|l|l|l|}
\hline Parameters & HR & 95\% CI & $\boldsymbol{p}$ values \\
\hline Interval time $(<13.63$ months vs. $\geq 13.63$ months) & 0.256 & $0.083-0.791$ & 0.018 \\
\hline PTV volume $(<15.33 \mathrm{~mL}$ vs. $\geq 15.33 \mathrm{~mL})$ & 3.656 & $1.348-9.913$ & 0.011 \\
\hline CT after SRT (yes vs. no) & 1.549 & $0.559-4.008$ & 0.367 \\
\hline
\end{tabular}

Abbreviations: OS: overall survival; NSCLC: non-small cell lung cancer; HR: hazard ratio; CI: confidence interval; SRT: stereotactic radiation therapy; PTV: planning target volume; CT: chemotherapy.

longer time from diagnosis to SRT), small disease burden (as suggested by smaller PTV) and improved patient performance status (as suggested by patients ability to receive chemotherapy after SRT) (Table 3). Thus, we believe that oligometastatic/oligorecurrent patients are a distinct subset of metastatic patients because many may be cured of their disease. Investigating novel curative local therapies that have minimal toxicity is important for these patients.

The low-toxicity profile (Table 4) observed in our study is of particular importance in cancer patients, who have received or will receive other oncologic therapies. The majority of our patients ( $>90 \%)$ experienced CTCAE Grade 1-2 acute or late toxic events; most of these symptoms were transient and resolved with conservative management. However, despite meeting normal tissue 
Table 4: Toxicities of patients with oligometastases or oligorecurrence to MLNs treated with SRT

\begin{tabular}{|l|l|l|l|l|}
\hline & Total, n (\%) & \multicolumn{4}{l|}{} \\
\hline Acute toxicities & Any Grade & Grade 3 & Grade 4 & Grade 5 \\
\hline Pneumonitis & $6(7)$ & $1(1)$ & 0 & 0 \\
\hline Esophagitis & $3(4)$ & $1(1)$ & 0 & 0 \\
\hline Tracheitis & $3(4)$ & $2(2)$ & 0 & 0 \\
\hline Chest pain & $1(1)$ & 0 & 0 & 0 \\
\hline Agranulocytosis & $1(1)$ & 0 & 0 & 0 \\
\hline Thrombocytopenia & $1(1)$ & 0 & 0 & 0 \\
\hline Late toxicities & & & & \\
\hline Tachycardia & $1(1)$ & 0 & 0 & 0 \\
\hline Lung fibrosis & $1(1)$ & 0 & 0 & 0 \\
\hline Atelectasis & $1(1)$ & 0 & 0 & 0 \\
\hline Tracheoesophageal fistula & $2(1)$ & 0 & 0 & $2(2)$ \\
\hline Esophageal-mediastinal fistula & $1(1)$ & 0 & 0 & $1(1)$ \\
\hline
\end{tabular}

Abbreviations: MLNs: mediastinal lymph nodes; SRT: stereotacticradiation therapy.
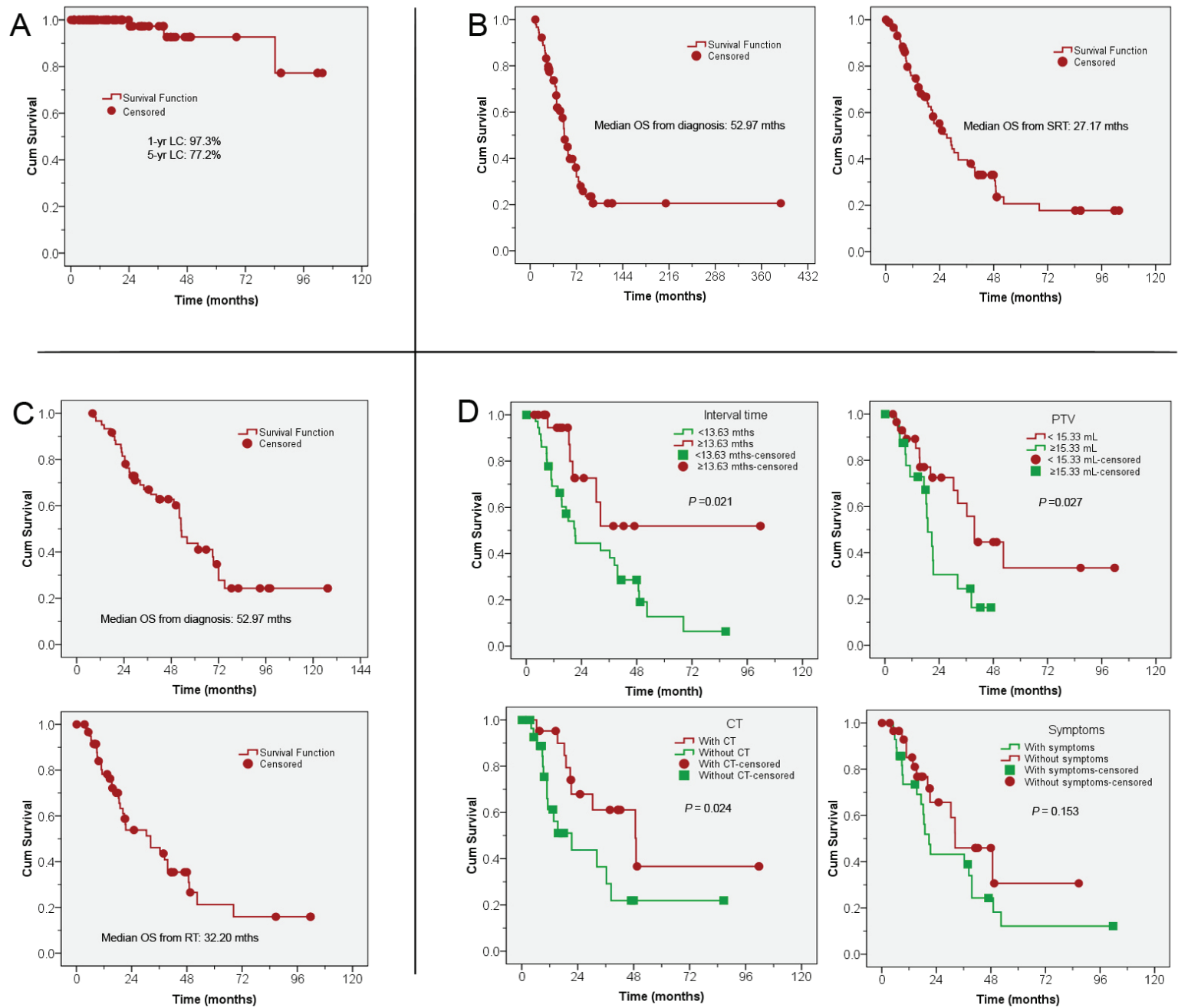

Figure 4: Actuarial LC and OS from time of diagnosis and from SRT for patients with oligometastases or oligorecurrence to MLNs. A. Actuarial LC for all patients; B. OS from diagnosis and from SRT for all patients; C. OS from diagnosis and from SRT for patients with NSCLC; D. OS for NSCLC patients by: interval time between diagnosis of the primary tumor and SRT (upper left); MLN PTV (upper right); presence or absence of CT (lower right); and presence or absence of symptoms (lower left). LC: local control; OS: overall survival; SRT: stereotactic radiation therapy; MLN: mediastinal lymph node; NSCLC: non-small cell lung cancer; PTV: planning target volume; CT: chemotherapy. 
constraints, three patients (4\%) died from Grade 5 late toxicities, all of which had history of RT to LN station 7. Our results corroborate those of Corradetti et al [35] nd we advise extreme caution when considering SRT (especially re-irradiation) around the mainstem bronchi (e.g. level 7 LNs), as this may cause central airway necrosis or tracheoesophageal fistula, as seen in the current study.

This study has limitations, including its retrospective nature, inclusion of a heterogeneous group of patients with different primary tumors, different MLN treatment sites, curative vs. palliative treatment intent, disease extent, RT history, fractionation regimens, and systemic therapies used. We can only draw associations but not causation between patient outcomes and treatment characteristics.

Further studies are needed to evaluate confounders, including age, comorbidity, performance status, histology, the primary tumor site, and genetic differences between the primary tumor and metastases. For example, certain patients may have a better performance status and may tolerate more systemic therapies (as seen in Figure 3). Thus, we recommend clinicians treat patients based on a personalized, multidisciplinary approach for each patient.

In conclusion, SRT is a safe and efficacious for patients with oligometastases or oligorecurrences to MLNs originating from different tumors, though we recommend caution in re-irradiation to MLN station 7. Palliation of symptoms is achievable in most patients with symptomatic lesions. Further investigation is warranted to identify the patients who benefit most from this treatment modality.

\section{PATIENTS AND METHODS}

\section{Study design and eligible patients}

We retrospectively queried our prospectivelycollected database of patients with oligometastases or oligorecurrence to a MLN, treated between October 1, 2006 and May 1, 2015. All patients were examined in a multidisciplinary setting at the time of diagnosis or recurrence, and their cases were re-presented to the tumor board as needed. The inclusion criteria were: (i) any age; (ii) Karnofsky performance score (KPS) $\geq 70$; (iii) oligometastasis or oligorecurrence to one or two MLNs within the MLN zone; (iv) any primary tumor site, with prior biopsy and histologic confirmation and either computed tomography (CT) or positron emission tomography (PET)-CT images; (v) life expectancy $\geq$ six months; (vi) unamenable to resection (either because of anatomical tumor characteristics or patient comorbidities); and (vii) patient written informed consent for the treatment and inclusion in the database. Exclusion criteria were contraindication for receiving $\mathrm{RT}$ and uncontrolled comorbid condition (metabolic or psychiatric). The study protocol was in accordance with the ethical guidelines of the 1995 Declaration of Helsinki and was approved by the independent ethics committees at Tianjin Medical University Cancer Institute \& Hospital, National Clinical Research Center for Cancer, China.

\section{Treatment}

MLN stations were classified according to Mountain and Dresler and were delineated following the atlas from the University of Michigan [36-37]. The methodology used for CyberKnife SRT and treatment planning was as described in our preliminary study [11]. Briefly, patients were immobilized using a vacuum bag before CT simulation. A set of planning three- and fourdimensional (3D/4D) CT images were obtained with IV contrast to highlight the MLN metastases. The gross target volume (GTV) was defined for the MLN disease based on simulation, CT, and/or PET-CT. The planning target volume (PTV) was defined as the GTV with a margin of $0.3 \mathrm{~cm}$. The PTV was also amended to avoid adjacent organs at risk (i.e. esophagus, brachial, trachea, spine cord, heart). The Xsight spine tracking system was used for positional alignment based on bony spinal skeletal structures.

BEDs were calculated based on the formula: $\operatorname{nd}[1$ $+d /(\alpha / \beta)$ ], where $n$ is number of fractions, and $d$ is dose/ fraction (Gy); assuming $\alpha / \beta$ value of 10 for lung cancer or acute toxicities (i.e. BED $_{10}$ ), and assuming $\alpha / \beta$ value of 3.0 for late toxicities (i.e. $\mathrm{BED}_{3}$ ). Our treating physicians typically try to increase dose of PTV $\mathrm{BED}_{10}$ to $>100 \mathrm{~Gy}$, as this has been shown to be associated with improved LC [38-41]. Unfortunately, delivery of a high BED to the tumor is not always possible, given the juxtaposition of PTV to critical structures (e.g. great vessels, esophagus). Moreover, for all patients with previous RT, the original treatment plans are incorporated and all BEDs were summed (in order to minimize the dose to critical organs), limiting the prescribed doses further. For normal tissues, we use constraints proposed by Kong et al, the Radiation Therapy Oncology Group (RTOG) 0236 and 0813 guidelines, and the NRG BR-001 guidelines (provided in Supplementary file 1) [42-44]. If patients received chemotherapy after SRT, data was gathered about the agents used and the number of cycles.

\section{Follow-up}

Patients were seen in the clinic at 1 month after completion of their treatment, then every 3 months for the first year, then every 6 months until May 1, 2015. Imaging, adverse events, and compliance of all patients were monitored during this follow-up period using our clinical databases. 


\section{Endpoints}

The primary end-point was local control rate (LC; defined as no progression of treated disease on follow-up scans), which was categorized as complete response (CR), partial response (PR), or stable disease (SD) using the RECIST 1.1 Response Evaluation Criteria in Solid Tumors [45]. Patients were considered to have a local failure if there was evidence of increased size of enhancing tumor in the treated region. PET-CT scan was employed to assist with differentiating radiation-related changes from local or regional recurrence. LC was assessed at a minimum of 6 months after SRT, in order to avoid uncertainty associated with early transient radiographic changes within the highdose region.

The secondary end-points were: (1) the time to symptom alleviation (defined as the time between the date of SRT completion and the date of symptom alleviation or the date of the last follow-up for censored patients); (2) overall survival (OS, defined as the time between the date of the SRT and the date of death or the date of the last follow-up for censored patients); (3) pattern of failure, including locoreginal failure and/or distant metastases; and (4) Common Terminology Criteria for Adverse Events (CTCAE v4.0) grade toxicity. All toxicities were assessed in a multidisciplinary setting.

\section{Statistical analysis}

LC and OS curves were estimated using KaplanMeier analysis and compared using the stratified logrank test, with $p$ value $\leq 0.05$ considered statistically significant. Data were analyzed using the statistical software Intercooled Stata version 8.2 for Windows (Stata Corporation, College Station, Texas, USA).

\section{ACKNOWLEDGMENTS}

This work was supported by the National Natural Science Foundation of China (No. 81201754), the New Teacher Fund for Doctor Station, the Ministry of Education (No. 20121202120014), the National Natural Science Foundation of China (No. 81472797), and the Foundation of National Clinical Research Center for Cancer (No. N14B04). No benefits in any form have been or will be received from a commercial party directly or indirectly related to the subject of this article. We also thank the anonymous reviewer for his/her very helpful comments, which improved the quality of this paper.

\section{CONFLICT OF INTEREST}

No benefits in any form have been or will be received from a commercial party directly or indirectly related to the subject of this article.

\section{Author contributions}

Conception and design: Meng MB.

Provision of study materials or patients: Meng MB, Wang HH, Zeng XL, Song YC, Zhuang HQ, Li FT, Yuan $\mathrm{ZY}$, and Wang P.

Collection and assembly of data: Meng MB, Wang HH, Zeng XL, Deng L, Zaorsky NG, Song YC, Zhuang HQ, Li FT, Zhao LJ, Yuan ZY, and Wang P.

Data analysis and interpretation: Meng MB, Wang HH, Zeng XL, Deng L, Zaorsky NG, Yuan ZY, and Wang P, Hao XS.

Manuscript writing: Meng MB, Wang HH, Deng L, and Zaorsky NG.

Final approval of manuscript: Meng MB, Wang $\mathrm{HH}$, Zeng XL, Deng L, Zaorsky NG, Song YC, Zhuang HQ, Li FT, Zhao LJ, Yuan ZY, and Wang P, Hao XS.

\section{REFERENCES}

1. Corbin KS, Hellman S, Weichselbaum RR. Extracranial oligometastases: a subset of metastases curable with stereotactic radiotherapy. J Clin Oncol 2013; 31: 13841390.

2. Casali C, Stefani A, Natali P, Rossi G, Morandi U. Prognostic factors in surgically resected N2 non-small cell lung cancer: the importance of patterns of mediastinal lymph nodes metastases. Eur J Cardiothorac Surg 2005; 28: 33-38.

3. Lo SS, Moffatt-Bruce SD, Dawson LA, et al. The role of local therapy in the management of lung and liver oligometastases. Nat Rev Clin Oncol 2011; 8: 405-416.

4. Palma DA, Salama JK, Lo SS, et al. The oligometastatic state-separating truth from wishful thinking. Nat Rev Clin Oncol 2014; 11: 549-557.

5. Timmerman RD, Kavanagh BD, Cho LC, Papiez L, Xing L. Stereotactic body radiation therapy in multiple organ sites. J Clin Oncol 2007; 25: 947-952.

6. Ben-Josef E, Lawrence TS. Using a bigger hammer: the role of stereotactic body radiotherapy in the management of oligometastases. J Clin Oncol 2009; 27: 1537-1539.

7. Tree AC, Khoo VS, Eeles RA, et al. Stereotactic body radiotherapy for oligometastases. Lancet Oncol 2013; 14: e28-e37.

8. Salama JK, Milano MT. Radical irradiation of extracranial oligometastases. J Clin Oncol 2014; 32: 2902-2912.

9. de Vin T, Engels B, Gevaert T, Storme G, De Ridder M. Stereotactic radiotherapy for oligometastatic cancer: a prognostic model for survival. Ann Oncol 2014; 25: 467471.

10. Nuyttens JJ, van der Voort van Zyp NC, Verhoef C, et al. Stereotactic body radiation therapy for oligometastases to the lung: a phase 2 study. Int J Radiat Oncol Biol Phys 2015; 91: 337-343. 
11. Meng MB, Wang HH, Zaorsky NG, et al. Clinical evaluation of stereotactic radiation therapy for recurrent or second primary mediastinal lymph node metastases originating from non-small cell lung cancer. Oncotarget 2015; 6: 15690-15703. doi: 10.18632/oncotarget.3704.

12. Trodella L, Trodella L, Granone P, et al. Adjuvant radiotherapy in non-small cell lung cancer with pathological stage I: definitive results of a phase III randomized trial. Radiother Oncol 2002; 62: 11-19.

13. Dautzenberg B, Arriagada R, Chammard AB, et al. A controlled study of postoperative radiotherapy for patients with completely resected nonsmall cell lung carcinoma. Groupe d'Etude et de Traitement des Cancers Bronchiques. Cancer 1999; 86: 265-273.

14. Kelly P, Balter PA, Rebueno N, et al. Stereotactic body radiation therapy for patients with lung cancer previously treated with thoracic radiation. Int J Radiat Oncol Biol Phys 2010; 78: 1387-1393.

15. Peulen H, Karlsson K, Lindberg K, et al. Toxicity after reirradiation of pulmonary tumors with stereotactic body radiotherapy. Radiother Oncol 2011; 101: 260-266.

16. Trakul N, Harris JP, Le QT, et al. Stereotactic ablative radiotherapy for reirradiation of locally recurrent lung tumors. J Thorac Oncol 2012; 7: 1462-1465.

17. Seung SK, Solhjem M. Salvage SBRT for previously irradiated lung cancer. J Cancer Ther 2011; 2: 190-195.

18. Kilburn JM, Kuremsky JG, Blackstock AW, et al. Thoracic re-irradiation using stereotactic body radiotherapy (SBRT) techniques as first or second course of treatment. Radiother Oncol 2014; 110: 505-510.

19. Jereczek-Fossa BA, Fariselli L, Beltramo G, et al. Linacbased or robotic image-guided stereotactic radiotherapy for isolated lymph node recurrent prostate cancer. Radiother Oncol 2009; 93: 14-17.

20. Choi CW, Cho CK, Yoo SY, et al. Image-guided stereotactic body radiation therapy in patients with isolated para-aortic lymph node metastases from uterine cervical and corpus cancer. Int J Radiat Oncol Biol Phys 2009; 74: 147-153.

21. Kim MS, Yoo SY, Cho CK, et al. Stereotactic body radiotherapy for isolated para-aortic lymph node recurrence after curative resection in gastric cancer. J Korean Med Sci 2009; 24: 488-492.

22. Kim MS, Cho CK, Yang KM, et al. Stereotactic body radiotherapy for isolated paraaortic lymph node recurrence from colorectal cancer. World J Gastroenterol 2009; 15: 6091-6095.

23. Kim JH, Kim MS, Yoo SY, et al. Stereotactic body radiotherapy for refractory cervical lymph node recurrence of nonanaplastic thyroid cancer. Otolaryngol Head Neck Surg 2010; 142: 338-343.

24. Bignardi M, Navarria P, Mancosu P, et al. Clinical outcome of hypofractionated stereotactic radiotherapy for abdominal lymph node metastases. Int J Radiat Oncol Biol Phys 2011;
81: 831-838.

25. Jereczek-Fossa BA, Beltramo G, Fariselli L, et al. Robotic image-guided stereotactic radiotherapy for isolated recurrent primary, lymph node or metastatic prostate cancer. Int J Radiat Oncol Biol Phys 2012; 82: 889-897.

26. Bae SH, Kim MS, Cho CK, et al. High dose stereotactic body radiotherapy using three fractions for colorectal oligometastases. J Surg Oncol 2012; 106: 138-143.

27. Jereczek-Fossa BA, Piperno G, Ronchi S, et al. Linac-based stereotactic body radiotherapy for oligometastatic patients with single abdominal lymph node recurrent cancer. Am J Clin Oncol 2014; 37: 227-233.

28. Rwigema JC, King C, Wang PC, et al. Stereotactic body radiation therapy for abdominal and pelvic oligometastases: dosimetric targets for safe and effective local control. Pract Radiat Oncol 2015; 5: 183-191.

29. Ponti E, Ingrosso G, Carosi A, et al. Salvage stereotactic body radiotherapy for patients with prostate cancer with isolated lymph node metastasis: A single-center experience. Clin Genitourin Cancer 2015; 13: e279-e284.

30. Kelsey CR, Clough RW, Marks LB. Local recurrence following initial resection of NSCLC: salvage is possible with radiation therapy. Cancer J 2006; 12: 283-288.

31. Kelsey CR, Clough RW, Marks LB. Local recurrence following initial resection of NSCLC: salvage is possible with radiation therapy. Cancer J 2006; 12: 283-288.

32. Belani CP, Choy H, Bonomi $\mathrm{P}$, et al. Combined chemoradiotherapy regimens of pacitaxel and carboplatin for locally advanced non-small cell lung cancer: a randomized phase II locally advanced multi-modality protocol. J Clin Oncol 2005; 23: 5883-5891.

33. Ournel P, Robinet G, Thomas P, et al. Randomized phase III trial of sequential chemoradiotherapy compared with concurrent chemoradiotherapy in locally advanced non-small-cell lung cancer: Groupe Lyon-Saint-Etienne d'Oncologie Thoracique-Groupe Français de PneumoCancérologie NPC 95-01 study. J Clin Oncol 2005; 23: 5910-5917.

34. Huber RM, Flentje M, Schmidt M, et al. Simultaneous chemoradiotherapy compared with radiotherapy alone after induction chemotherapy in inoperable stage IIIA or IIIB non-small-cell lung cancer: study CTRT99/97 by the Bronchial Carcinoma Therapy Group. J Clin Oncol 2006; 24: 4397-4404.

35. Corradetti MN, Haas AR, Rengan R. Central-airway necrosis after stereotactic body-radiation therapy. N Engl J Med 2012; 366: 2327-2329.

36. Mountain CF, Dresler CM. Regional lymph node classification for lung cancer staging. Chest 1997; 111: 1718-1723.

37. Kong FM, Ritter T, Quint DJ, et al. Consideration of dose limits for organs at risk of thoracic radiotherapy: atlas for lung, proximal bronchial tree, esophagus, spinal cord, ribs, and brachial plexus. Int J Radiat Oncol Biol Phys 2011; 81: 
1442-1457.

38. Guckenberger M, Wulf J, Mueller G, et al. Doseresponse relationship for image-guided stereotactic body radiotherapy of pulmonary tumors: relevance of 4D dose calculation. Int J Radiat Oncol Biol Phys 2009; 74: 47-54.

39. Hiraoka M, Nagata Y. Stereotactic body radiation therapy for early-stage non-small-cell lung cancer: the Japanses experience. Int J Clin Oncol 2004; 9: 352-355.

40. Zaorsky NG, Palmer JD, Hurwitz MD, Keith SW, Dicker $\mathrm{AP}$, Den RB. What is the ideal radiotherapy dose to treat prostate cancer? A meta-analysis of biologically equivalent dose escalation. Radiother Oncol 2015; 115: 395-300.

41. Senthi S, Haasbeek CJ, Slotman BJ, Senan S. Outcomes of stereotactic ablative radiotherapy for central lung tumors: a systematic review. Radiother Oncol 2013; 106: 276-282.
42. Timmerman R, Paulus R, Galvin J, et al. Stereotactic body radiation therapy for inoperable early stage lung cancer. JAMA 2010; 303: 1070-1076.

43. Radiation Therapy Oncology Group. RTOG 0813: Seamless phase I/II study of stereotactic lung radiotherapy (SBRT) for early stage, centrally located, non-small cell lung cancer (NSCLC) in medically inoperable patients. Available at http://www.rtog.org/ClinicalTrials/ProtocolTable/ StudyDetails.aspx?study $=0813$.

44. NRG Oncology. A phase 1 study of stereotactic body radiotherapy (SBRT) for the treatment of multiple metastases. Available at https://clinicaltrials.gov/ct2/show/ NCT02206334?term=NRG-BR001\&rank=1.

45. Eisenhauer EA, Therasse P, Bogaerts J, et al. New response evaluation criteria in solid tumors: revised RECIST guideline (version 1.1). Eur J Cancer 2009; 45: 228-247. 